\title{
RTK-VPPP Algorithms in Urban Canyons
}

\author{
Sueo Sugimoto, Youta Suzuki and Yukihiro Kubo \\ Department of Electrical and Electronic Engineering, Ritsumeikan University \\ 1-1-1 Noji-Higashi, Kusatsu City, Shiga 525-8577 Japan \\ Tel: +81-(0)77-561-2673, FAX: +81-(0)77-561-2663 \\ E-mail: sugimoto@se.ritsumei.ac.jp
}

\begin{abstract}
In this paper we present a carrier-phase-based RTK-PPP algorithm with multiple antennas which are disposed with solid geometrical distances and with the common receivers' clock errors, based on GR models. We present GR equations for multiple antennas, and derive the Kalman filtering formula of very precise point positioning in urban cayons.
\end{abstract}

\section{INTRODUCTION}

In this paper we present the carrier-phase-based Real Time Kinematic - Precise Point Positioning (RTK-PPP) algorithms with multiple antennas [1]-[3] in urban cayons [4][6] based on Global navigation satellite system Regression models (referred as GR models) which have been developed in [7]-[13].

Precise point positioning (PPP) is a ultimately desirable technology in the GPS/GNSS positioning community [14]. Our proposed positioning algorithm achieve the positioning accuracy in decimeter level [9]-[11] without any external transmitted information such as from the wide area augmentation system (WAAS).

According to $[12,13]$, in this paper, we present GR equations which contain the so-called receiver's delay such that very precise point positioning (VPPP) algorithms derived by using multiple antennas which are disposed with solid geometrical distances and with the common receivers' clock errors. The measurements data are obtained from L1, L2 band carrier phases as well as the pseudoranges based on $\mathrm{C} / \mathrm{A}$ and $\mathrm{P}(\mathrm{Y})$ codes, respectively, and the regression matrix in GR models is given by computing the gradient vector of the geometric distance between the receiver $u$ and the satellite $s$ with respect to the $\nu$-th estimated value $\hat{u}^{(\nu)}$ and $\hat{s}$.

In the next section, we show the GR models and use them to derive a PPP algorithm. In the section 3, the PPP algorithm is extended to VPPP using two or more GNSS antennas with common clock errors and the known distances among receivers [1]-[3]. Then we develop two types of estimation algorithms (Methods 1 and 2) for using the constraints of the distances among antennas, based on the recursive Kalman filtering. These positioning algorithms may give very accurate positioning results than those in only one antenna due to the constraints of the antennas' positions and common receivers' clock errors [1]-[3].

\section{GNSS REGRESSION MODELS}

Similarly to [7]-[13], we formulate all observed positioning data consisting of L1 and L2 carrier phases and pseudoranges based on C/A code, if available, and also $\mathrm{P}(\mathrm{Y})$ code, by using novel new GNSS regression models. The natural extensions of GNSS regression models for multiple frequencies of Galileo and US-GPS modernization are also similarly formulated. Namely, we consider the following fundamental measurements of $L_{1}, L_{2}$ band carrier phases $\varphi_{L 1, u}^{p}(t), \varphi_{L 2, u}^{p}(t)$; and the pseudoranges $\rho_{C A, u}^{p}(t), \rho_{P Y, u}^{p}(t)$ based on $\mathrm{C} / \mathrm{A}$ and $\mathrm{P}(\mathrm{Y})$ codes, respectively, as follows [15], [16]-[19]:

$$
\begin{aligned}
\rho_{C A, u}^{p}(t)= & r_{u}^{p}\left(t, t-\tau_{u}^{p}\right)+\delta I_{u}^{p}(t)+\delta T_{u}^{p}(t) \\
& +c\left[\delta t_{u}(t)-\delta t^{p}\left(t-\tau_{u}^{p}\right)\right] \\
& +\delta b_{C A, u}-\delta b_{C A}^{p}+e_{C A, u}^{p}(t) \\
\rho_{P Y, u}^{p}(t)= & r_{u}^{p}\left(t, t-\tau_{u}^{p}\right)+\frac{f_{1}^{2}}{f_{2}^{2}} \delta I_{u}^{p}(t)+\delta T_{u}^{p}(t) \\
& +c\left[\delta t_{u}(t)-\delta t^{p}\left(t-\tau_{u}^{p}\right)\right] \\
& +\delta b_{P Y, u}-\delta b_{P Y}^{p}+e_{P Y, u}^{p}(t), \\
\Phi_{L 1, u}^{p}(t)= & \lambda_{1} \varphi_{L 1, u}^{p}(t) \\
= & r_{u}^{p}\left(t, t-\tau_{u}^{p}\right)-\delta I_{u}^{p}(t)+\delta T_{u}^{p}(t) \\
& +c\left[\delta t_{u}(t)-\delta t^{p}\left(t-\tau_{u}^{p}\right)\right] \\
& +\delta b_{L 1, u}-\delta b_{L 1}^{p}+\lambda_{1} N_{u}^{p}+\lambda_{1} \varepsilon_{u}^{p}(t) \\
\Phi_{L 2, u}^{p}(t)= & \lambda_{2} \varphi_{L 2, u}^{p}(t) \\
= & r_{u}^{p}\left(t, t-\tau_{u}^{p}\right)-\frac{f_{1}^{2}}{f_{2}^{2}} \delta I_{u}^{p}(t)+\delta T_{u}^{p}(t) \\
& +c\left[\delta t_{u}(t)-\delta t^{p}\left(t-\tau_{u}^{p}\right)\right] \\
& +\delta b_{L 2, u}-\delta b_{L 2}^{p}+\lambda_{2} N_{u}^{p}+\lambda_{2} \varepsilon_{u}^{p}(t)
\end{aligned}
$$

where $c\left(\approx 2.99792458 \times 10^{8}[\mathrm{~m}]\right)$ denotes the speed of light, and $f_{i}$ and $\lambda_{i}$ are the central frequency and the wave length of the $L_{i}$ carrier wave

$$
\begin{aligned}
& f_{1}=2 \times 77 \times 10.23[\mathrm{MHz}]=1575.42[\mathrm{MHz}], \\
& f_{2}=2 \times 60 \times 10.23[\mathrm{MHz}]=1227.60[\mathrm{MHz}] .(5)
\end{aligned}
$$

In (1)-(4), the so-called receiver's biases, $\left\{\delta b_{C A, u}, \delta b_{P Y, u}\right.$, $\left.\delta b_{L 1, u}, \delta b_{L 2, u}\right\}$, and the satellite biases, $\left\{\delta b_{C A}^{p}, \delta b_{P Y}^{p}, \delta b_{L 1}^{p}\right.$, $\left.\delta b_{L 2}^{p}\right\}$, are contained in the usual observed positioning data 
consisting of L1 and L2 carrier phases and pseudoranges based on C/A and $\mathrm{P}(\mathrm{Y})$ codes [14]. Also $r_{u}^{p}\left(t, t-\tau_{u}^{p}\right)$ is the geometric distance between the receiver $u$ at the time $t$ and the satellite $p$ at the time $t-\tau_{u}^{p}$ ( $\tau_{u}^{p}$ denotes the travel time from the satellite $p\left(p=1, \ldots, n_{s}\right)$ to the receiver $\left.u\right)$. Namely,

$$
\begin{aligned}
r_{u}^{p}(t) \equiv & r_{u}^{p}\left(t, t-\tau_{u}^{p}\right) \\
= & {\left[\left(x_{u}(t)-x^{p}\left(t-\tau_{u}^{p}\right)\right)^{2}+\left(y_{u}(t)-y^{p}\left(t-\tau_{u}^{p}\right)\right)^{2}\right.} \\
& \left.+\left(z_{u}(t)-z^{p}\left(t-\tau_{u}^{p}\right)\right)^{2}\right]^{1 / 2} \\
= & \left\|u(t)-s^{p}\left(t-\tau_{u}^{p}\right)\right\|
\end{aligned}
$$

where $u \equiv\left[x_{u}, y_{u}, z_{u}\right]^{\mathrm{T}}$ and $s^{p} \equiv\left[x^{p}, y^{p}, z^{p}\right]^{\mathrm{T}}$ are a user (unknown) and satellite positions, respectively. Also $n_{s}$ shows the number of the observable satellites. Further in (1)-(4), $\delta I_{u}^{p}(t)$ and $\delta T_{u}^{p}(t)$ reflect the delay or the advance associated with the transmission of the $L_{1}$ signal through the ionosphere and the troposphere, respectively. $\delta t_{u}(t)$ and $\delta t^{p}\left(t-\tau_{u}^{p}\right)$ are the clock errors of the receiver $u$ at the time $t$ and the satellite $p$ at the time $t-\tau_{u}^{p}$. $N_{u}^{p}$ denotes integer ambiguity between the satellite $p$ and the receiver $u$, and $\varepsilon^{p}(t), e^{p}(t)$ denote measurement errors.

Eq. (6) contains the satellite orbital errors. The estimated satellite orbits are obtained from the navigation messages which are decoded from the transmitted $L 1$ signal. Let us denote $\hat{s}^{p}$ as the estimated position of the satellite $s^{p}$ at the time $t-\tau_{u}^{p}$.

We use the following relations of the derivatives

$$
\begin{array}{ll}
\frac{\partial r_{u}^{p}}{\partial x_{u}}=\frac{\left(x_{u}-x^{p}\right)}{r_{u}^{p}}, & \frac{\partial r_{u}^{p}}{\partial y_{u}}=\frac{\left(y_{u}-y^{p}\right)}{r_{u}^{p}}, \\
\frac{\partial r_{u}^{p}}{\partial z_{u}}=\frac{\left(z_{u}-z^{p}\right)}{r_{u}^{p}}, & \left(p=1,2, \ldots, n_{s}\right),
\end{array}
$$

and also

$$
\begin{array}{ll}
\frac{\partial r_{u}^{p}}{\partial x^{p}}=-\frac{\left(x_{u}-x^{p}\right)}{r_{u}^{p}}, & \frac{\partial r_{u}^{p}}{\partial y^{p}}=-\frac{\left(y_{u}-y^{p}\right)}{r_{u}^{p}}, \\
\frac{\partial r_{u}^{p}}{\partial z^{p}}=-\frac{\left(z_{u}-z^{p}\right)}{r_{u}^{p}}, & \left(p=1,2, \ldots, n_{s}\right) .
\end{array}
$$

Then we have the relation:

$$
\frac{\partial r_{u}^{p}}{\partial u}=-\frac{\partial r_{u}^{p}}{\partial s^{p}}
$$

Thus the 1st order Taylor series approximation of (6) around the previous estimated value $u=\hat{u}^{(\nu)}$ and $s^{p}=\hat{s}^{p}$ is given by

$$
\begin{aligned}
r_{u}^{p} & \cong r_{\hat{u}^{(\nu)}}^{\hat{p}}+g_{\hat{u}^{(\nu)}}^{\hat{p}}\left[u-s^{p}-\left(\hat{u}^{(\nu)}-\hat{s}^{p}\right)\right] \\
& =\left\|\hat{u}^{(\nu)}-\hat{s}^{p}\right\|+\frac{\left(\hat{u}^{(\nu)}-\hat{s}^{p}\right)^{\mathrm{T}}}{\left\|\hat{u}^{(\nu)}-\hat{s}^{p}\right\|}\left[u-s^{p}-\left(\hat{u}^{(\nu)}-\hat{s}^{p}\right)\right] \\
& =\frac{\left(\hat{u}^{(\nu)}-\hat{s}^{p}\right)^{\mathrm{T}}}{\left\|\hat{u}^{(\nu)}-\hat{s}^{p}\right\|}\left(u-s^{p}\right)
\end{aligned}
$$

for $p=1,2, \ldots, n_{s}$, where

$$
g_{\hat{u}^{(\nu)}}^{\hat{p}} \equiv\left[\frac{\partial r_{u}^{p}}{\partial u}\right]_{u=\hat{u}^{(\nu)}, s^{p}=\hat{s}^{p}}^{\mathrm{T}}=\frac{\left(\hat{u}^{(\nu)}-\hat{s}^{p}\right)^{\mathrm{T}}}{\left\|\hat{u}^{(\nu)}-\hat{s}^{p}\right\|}
$$

From (1)-(4), therefore, we have the approximations:

$$
\begin{aligned}
\rho_{C A, u}^{p} \cong & g_{\hat{u}^{(\nu)}}^{\hat{p}}\left(u-s^{p}\right) \\
& +\delta I_{u}^{p}+\delta T_{u}^{p}+c\left(\delta t_{u}-\delta t^{p}\right) \\
& +\delta b_{C A, u}-\delta b_{C A}^{p}+e_{C A, u}^{p}, \\
\rho_{P Y, u}^{p} \cong & g_{\hat{u}^{(\nu)}}^{\hat{p}}\left(u-s^{p}\right) \\
& +\frac{f_{1}^{2}}{f_{2}^{2}} \delta I_{u}^{p}(t)+\delta T_{u}^{p}+c\left(\delta t_{u}-\delta t^{p}\right) \\
& +\delta b_{P Y, u}-\delta b_{P Y}^{p}+e_{P Y, u}^{p}, \\
\cong & g_{\hat{u}^{(\nu)}}^{\hat{p}}\left(u-s^{p}\right) \\
& -\delta I_{u}^{p}+\delta T_{u}^{p}+c\left(\delta t_{u}-\delta t^{p}\right) \\
& +\delta b_{L 1, u}-\delta b_{L 1}^{p}+\lambda_{1} N_{L 1, u}^{p}+\lambda_{1} \varepsilon_{L 1, u}^{p}, \\
\Phi_{L 1, u}^{p}, & g_{\hat{u}^{(\nu)}}^{\hat{u}^{(\nu)}}\left(u-s^{p}\right) \\
& +\frac{f_{1}^{2}}{f_{2}^{2}} \delta I_{u}^{p}+\delta T_{u}^{p}+c\left(\delta t_{u}-\delta t^{p}\right) \\
& +\delta b_{L 2, u}-\delta b_{L 2}^{p}+\lambda_{2} N_{L 2, u}^{p}+\lambda_{2} \varepsilon_{L 2, u}^{p} .
\end{aligned}
$$

Define the $n_{s} \times 3$ matrix:

$$
G_{\hat{u}^{(\nu)}}^{\hat{s}} \equiv\left[\begin{array}{c}
g_{\hat{u}^{(\nu)}}^{\hat{1}} \\
g_{\hat{u}^{(\nu)}}^{2} \\
\vdots \\
g_{\hat{u}^{(\nu)}}^{\hat{n}_{s}}
\end{array}\right],
$$

namely,

$$
G_{\hat{u}^{(\nu)}}^{\hat{s}}=\left[\begin{array}{ccc}
\frac{\partial r_{\hat{u}^{(\nu)}}^{\hat{1}^{(\nu)}}}{\partial \hat{x}_{u}^{(\nu)}} & \frac{\partial r_{\hat{u}^{(\nu)}}^{\hat{1}}}{\partial \hat{y}_{u}^{(\nu)}} & \frac{\partial r_{\hat{u}^{(\nu)}}^{\hat{u}^{(\nu)}}}{\partial \hat{z}_{u}^{(\nu)}} \\
\frac{\partial r_{\hat{u}^{(\nu)}}^{\hat{2}}}{\partial \hat{x}_{u}^{(\nu)}} & \frac{\partial r_{\hat{u}^{(\nu)}}^{\hat{2}}}{\partial \hat{y}_{u}^{(\nu)}} & \frac{\partial r_{\hat{u}^{(\nu)}}^{\hat{2}}}{\partial \hat{z}_{u}^{(\nu)}} \\
\vdots & \vdots & \vdots \\
\frac{\partial r_{\hat{u}_{s}^{(\nu)}}^{\hat{n}_{s}}}{\partial \hat{x}_{u}^{(\nu)}} & \frac{\partial r_{\hat{u}^{(\nu)}}^{\hat{n}_{s}}}{\partial \hat{y}_{u}^{(\nu)}} & \frac{\partial r_{\hat{u}_{s}^{(\nu)}}^{\hat{n}_{s}}}{\partial \hat{z}_{u}^{(\nu)}}
\end{array}\right] .
$$

Now let us discuss the satellite's as well as receiver's hardware biases $\delta b^{p}$, and $\delta b_{*, u}$. It was pointed out in [15] that the magnitudes of the satellite's hardware biases are usually in the range of (several nanosecond $\times c$ ) while the receiver's hardware biases could exceed 10 nanoseconds $\times c$. Therefore, we assume that the satellite's hardware biases may be negligible. On the other hand, we do not disregard the receiver's hardware biases such that we define the terms the $4 \times 1$ vector

$$
\delta b_{u} \equiv\left[\delta b_{C A, u}, \delta b_{P Y, u}, \delta b_{L 1, u}, \delta b_{L 2, u}\right]^{\mathrm{T}} .
$$


Furthermore, from (16), we define a block diagonal matrix with the size $\left(n_{s} \times 3 n_{s}\right)$ :

$$
G_{D, \hat{u}(\nu)}^{\hat{s}} \equiv\left[\begin{array}{ccccc}
g_{\hat{u}^{(\nu)}}^{\hat{1}} & O & O & \cdots & O \\
O & g_{\hat{u}^{(\nu)}}^{\hat{2}} & O & \cdots & O \\
\vdots & & \ddots & & \vdots \\
\vdots & & & \ddots & O \\
O & \cdots & \cdots & O & g_{\hat{u}_{s}^{(\nu)}}^{\hat{n}_{s}}
\end{array}\right] .
$$

Then from (12)-(19), we have the following vector matrix regression equation:

$$
y_{\hat{u}^{(\nu)}}=H_{\hat{u}^{(\nu)}} \theta_{u}+v_{u},
$$

where

$$
\begin{aligned}
& y_{\hat{u}^{(\nu)}} \equiv\left[\begin{array}{c}
\rho_{C A, \hat{u}^{(\nu)}} \\
\rho_{P Y, \hat{u}^{(\nu)}} \\
\Phi_{L 1, \hat{u}^{(\nu)}} \\
\Phi_{L 2, \hat{u}^{(\nu)}}
\end{array}\right], \quad \theta_{u} \equiv\left[\begin{array}{c}
u \\
c \delta t_{u} \\
\delta b_{u} \\
s \\
c \delta t^{s} \\
\delta I_{u} \\
\delta T_{u} \\
\lambda_{1} N_{L 1, u} \\
\lambda_{2} N_{L 2, u}
\end{array}\right] \\
& H_{\hat{u}^{(\nu)}} \equiv\left[\begin{array}{ccccccccc}
G_{\hat{u}^{(\nu)}}^{\hat{s}} & \mathbf{1} & \mathbf{1} & -G_{D, \hat{u}^{(\nu)}}^{\hat{s}} & -I & I & I & O & O \\
G_{\hat{u}^{(\nu)}}^{\hat{s}} & \mathbf{1} & \mathbf{1} & -G_{D, \hat{u}^{(\nu)}}^{\hat{s}} & -I & \frac{f_{1}^{2}}{f_{2}^{2}} I & I & O & O \\
G_{\hat{x}^{(\nu)}}^{\hat{s}} & \mathbf{1} & \mathbf{1} & -G_{D, \hat{u}^{(\nu)}}^{\hat{s}} & -I & -I & I & I & O \\
G_{\hat{u}^{(\nu)}}^{\hat{s}} & \mathbf{1} & \mathbf{1} & -G_{D, \hat{u}^{(\nu)}}^{\hat{s}} & -I & -\frac{f_{1}^{2}}{f_{2}^{2}} I & I & O & I
\end{array}\right] \\
& v_{u} \equiv\left[e_{C A, u}^{\mathrm{T}}, e_{P Y, u}^{\mathrm{T}}, \lambda_{1} \varepsilon_{L 1, u}^{\mathrm{T}}, \lambda_{2} \varepsilon_{L 2, u}^{\mathrm{T}}\right]^{\mathrm{T}},
\end{aligned}
$$

and $I$ denote the $n_{s} \times n_{s}$ unit matrix and $\mathbf{1} \equiv[1,1, \cdots, 1]^{\mathrm{T}}$ : $n_{s} \times 1$ vector.

Then we get some knowledge of the satellite position $s$, the satellite clock error $c \delta t^{s}$ as well as the delay or the advance due to the ionospheric and tropospheric effects, $\delta I_{u}^{\mathrm{T}}$ and $\delta T_{u}^{\mathrm{T}}$ are, for instance,

$$
\begin{aligned}
\tilde{s} & =s+e_{s}, \\
c \tilde{\delta} t^{s} & =c \delta t^{s}+e_{\delta t^{s}}, \\
\tilde{\delta I_{u}} & =\delta I_{u}+e_{\delta I_{u}}, \\
\tilde{\delta T_{u}} & =\delta T_{u}+e_{\delta T_{u}} .
\end{aligned}
$$

Substituting the above relations into the GR equation (20), and neglect $s, c \delta t^{s}, \delta I_{u}, \delta T_{u}$, we have

$$
\left[\begin{array}{c}
y_{C \hat{s} A, \hat{u}^{(\nu)}}^{\hat{s}} \\
y_{P Y, \hat{u}^{(\nu)}}^{\hat{s}} \\
y_{L 1, \hat{u}^{(\nu)}}^{\hat{s}} \\
y_{L 2, \hat{u}^{(\nu)}}^{\hat{s}}
\end{array}\right]=C_{\hat{u}^{(\nu)}}^{\hat{s}}\left[\begin{array}{c}
u \\
c \delta t_{u} \\
\delta b_{u} \\
\lambda_{1} N_{L 1, u} \\
\lambda_{2} N_{L 2, u}
\end{array}\right]+v
$$

where

$$
\begin{aligned}
& C_{\hat{u}(\nu)}^{\hat{s}}=\left[\begin{array}{lllllllll}
G_{\hat{u}}^{\hat{s}} & \mathbf{1} & \mathbf{1} & & & & O & O \\
G_{\hat{u}}^{\hat{s}} & \mathbf{1} & & \mathbf{1} & & & O & O \\
G_{\hat{u}(\nu)}^{\hat{s}} & \mathbf{1} & & & \mathbf{1} & & I & O \\
G_{\hat{u}^{(\nu)}}^{\hat{s}} & \mathbf{1} & & & & \mathbf{1} & O & I
\end{array}\right], \\
& v=\left[\begin{array}{c}
G_{D, \hat{u}^{(\nu)}}^{\hat{s}} e_{s}+e_{\delta t^{s}}-e_{\delta I_{u}}-e_{\delta T_{u}}+e_{C A, u} \\
G_{D, \hat{u}^{(\nu)}}^{\hat{s}} e_{s}+e_{\delta t^{s}}-\frac{f_{1}^{2}}{f_{2}^{2}} e_{\delta I_{u}}-e_{\delta T_{u}}+e_{P Y, u} \\
G_{D, \hat{u}^{(\nu)}}^{\hat{s}} e_{s}+e_{\delta t^{s}}+e_{\delta I_{u}}-e_{\delta T_{u}}+\lambda_{1} \varepsilon_{L 1, u} \\
G_{D, \hat{u}^{(\nu)}}^{\hat{s}} e_{s}+e_{\delta t^{s}}+\frac{f_{1}^{2}}{f_{2}^{2}} e_{\delta I_{u}}-e_{\delta T_{u}}+\lambda_{2} \varepsilon_{L 2, u}
\end{array}\right],
\end{aligned}
$$

and

$$
\begin{aligned}
& y_{C A, \hat{u}^{(\nu)}}^{\hat{s}}=\rho_{C A, u}^{s}+G_{D, \hat{u}^{(\nu)}}^{\hat{s}} \tilde{s}+\tilde{c} \tilde{\delta} t^{s}-\tilde{\delta I}_{u}-\tilde{\delta T_{u}}, \quad \text { (30) } \\
& y_{P Y, \hat{u}^{(\nu)}}^{\hat{s}}=\rho_{P Y, u}^{s}+G_{D, \hat{u}^{(\nu)}}^{\hat{s}} \tilde{s}+c \tilde{\delta} t^{s}-\frac{f_{1}^{2}}{f_{2}^{2}} \tilde{\delta I_{u}}-\tilde{\delta T} \tilde{T}_{u}(31) \\
& y_{L 1, \hat{u}^{(\nu)}}^{\hat{s}}=\Phi_{L 1, u}^{s}+G_{D, \hat{u}^{(\nu)}}^{\hat{s}} \tilde{s}+c \tilde{\delta} t^{s}+\tilde{\delta I}_{u}-\tilde{\delta} \tilde{T}_{u}, \quad \text { (32) } \\
& y_{L 2, \hat{u}^{(\nu)}}^{\hat{s}}=\Phi_{L 2, u}^{s}+G_{D, \hat{u}^{(\nu)}}^{\hat{s}} \tilde{s}+c \tilde{\delta} t^{s}+\frac{f_{1}^{2}}{f_{2}^{2}} \tilde{\delta I}_{u}-\tilde{\delta T} \tilde{T}_{u}(33)
\end{aligned}
$$

\section{VERY PRECISE POINT POSITIONING}

Hereafter, we only describe the case of single frequency GR models due to simpler description and the commercial usage of postioning. Namely, we have the GR equations for L1 frequency as follows:

$$
\begin{aligned}
{\left[\begin{array}{c}
y_{C A, \hat{u}^{(\nu)}}^{\hat{s}} \\
y_{L 1, \hat{u}^{(\nu)}}^{\hat{s}}
\end{array}\right] } & =C_{\hat{u}^{(\nu)}}^{\hat{s}}\left[\begin{array}{c}
u \\
c \delta t_{u} \\
\delta b_{u} \\
\lambda_{1} N_{L 1, u}
\end{array}\right]+v_{u} \\
& =\tilde{C}_{\hat{u}^{(\nu)}}^{\hat{s}}\left[\begin{array}{c}
c \delta t_{u} \\
u \\
\delta b_{u} \\
\lambda_{1} N_{L 1, u}
\end{array}\right]+v_{u}
\end{aligned}
$$

where $\delta b_{u}=\left[\begin{array}{ll}\delta_{C A, u}, & \delta_{L 1, u}\end{array}\right]^{\mathrm{T}}$,

$$
\begin{aligned}
\tilde{C}_{\hat{u}(\nu)}^{\hat{s}} & =\left[\begin{array}{llll}
\mathbf{1} & G_{\hat{u}^{(\nu)}}^{\hat{s}} & \mathbf{1} & \mathbf{1} \\
\mathbf{1} & G_{\hat{u}^{(\nu)}}^{s} & \mathbf{1} & I
\end{array}\right] \\
v_{u} & =\left[\begin{array}{c}
G_{D, \hat{u}^{(\nu)}}^{\hat{s}} e_{s}+e_{\delta t^{s}}-e_{\delta I_{u}}-e_{\delta T_{u}}+e_{C A, u} \\
G_{D, \hat{u}^{(\nu)}}^{\hat{s}} e_{s}+e_{\delta t^{s}}+e_{\delta I_{u}}-e_{\delta T_{u}}+\lambda_{1} \varepsilon_{L 1, u}
\end{array}\right],
\end{aligned}
$$

and

$$
\begin{aligned}
y_{C A, \hat{u}^{(\nu)}}^{\hat{s}} & =\rho_{C A, u}^{s}+G_{D, \hat{u}^{(\nu)}}^{\hat{s}} \tilde{s}+c \tilde{\delta} t^{s}-\tilde{\delta I} \tilde{I}_{u}-\tilde{\delta T} \tilde{T}_{u},(36) \\
y_{L 1, \hat{u}^{(\nu)}}^{\hat{s}} & =\Phi_{L 1, u}^{s}+G_{D, \hat{u}^{(\nu)}}^{\hat{s}} \tilde{s}+c \tilde{\delta} t^{s}+\tilde{\delta} I_{u}-\tilde{\delta T_{u}} \cdot(37)
\end{aligned}
$$

Now let us consider that multiple antennas $u_{i}, i=1, \ldots, n_{r}$ are disposed with the given distance $d_{i, j}(\leq$ several [m]) between any two antennas $u_{i}$ and $u_{j}$. In this assumption, we 
have the following $n_{r}$ single frequency GR equations:

$$
\begin{aligned}
y_{\hat{u}_{1}^{(\nu)}} & =\tilde{C}_{\hat{u}^{(\nu)}}^{\hat{s}} \theta_{u_{1}}+v_{u_{1}}, \\
& \vdots \\
y_{\hat{u}_{n_{r}}^{(\nu)}}= & \tilde{C}_{\hat{u}^{(\nu)}}^{\hat{s}} \theta_{u_{n_{r}}}+v_{u_{n_{r}}},
\end{aligned}
$$

with the constraint

$$
\begin{aligned}
d_{i, j}= & \left\|u_{i}-u_{j}\right\|+\text { small noise } \\
=\sqrt{\left(x_{i}-x_{j}\right)^{2}+\left(y_{i}-y_{j}\right)^{2}+\left(z_{i}-z_{j}\right)^{2}} & + \text { small noise }
\end{aligned}
$$

The constraint can be linearized by applying the first order Taylor's expansion around the estimates $\hat{u}_{i}^{(\nu)}$ and $\hat{u}_{j}^{(\nu)}$ of $u_{i}$ and $u_{j}$, respectively, namely,

$$
d_{i, j}=\left\|u_{i}-u_{j}\right\| \approx \frac{\left(\hat{u}_{i}^{(\nu)}-\hat{u}_{j}^{(\nu)}\right)^{\mathrm{T}}}{\left\|\hat{u}_{i}^{(\nu)}-\hat{u}_{j}^{(\nu)}\right\|}\left(u_{i}-u_{j}\right)+v_{i j . d}
$$

Now we remark the common components appeared in $\theta_{u_{1}}$ to $\theta_{u_{n_{r}}}$ such that we combine all GR equations (38) - (39),

we have

$$
\left[\begin{array}{c}
y_{C A, \hat{u}_{1}^{(\nu)}}^{\hat{s}} \\
y_{L 1, \hat{u}_{1}^{(\nu)}}^{\hat{s}} \\
\vdots \\
y_{C A, \hat{u}_{n_{r}}^{(\nu)}}^{\hat{s}} \\
y_{L 1, \hat{u}_{n_{r}}^{(\nu)}}^{(\nu)}
\end{array}\right]=\tilde{C}_{\hat{u}_{1} \cdots \hat{n}_{r}}\left[\begin{array}{c}
c \delta t_{u} \\
u_{1} \\
\delta b_{u_{1}} \\
\lambda_{1} N_{L 1, u_{1}} \\
\vdots \\
u_{n_{r}} \\
\delta b_{u_{n_{r}}} \\
\lambda_{1} N_{L 1, u_{n_{r}}}
\end{array}\right]+v
$$

where

$$
\begin{gathered}
y_{C A, \hat{u}_{1}^{(\nu)}}^{\hat{s}}=\rho_{C A, \hat{u}_{1}^{(\nu)}}^{\hat{s}}+G_{D, \hat{u}_{1}^{(\nu)}}^{\hat{s}} \tilde{s}+c \tilde{\delta} t^{s}-\tilde{\delta} I_{u}-\delta \tilde{T}_{u}, \\
y_{L 1, \hat{u}_{1}^{(\nu)}}^{\hat{s}}=\rho_{L 1, \hat{u}_{1}^{(\nu)}}^{\hat{s}}+G_{D, \hat{u}_{1}^{(\nu)}}^{\hat{s}} \tilde{s}+c \tilde{\delta} t^{s}+\tilde{\delta} I_{u}-\delta \tilde{T}_{u}, \\
\vdots \\
y_{C A, \hat{u}_{n_{r}}^{(\nu)}}^{\hat{s}}=\rho_{C A, \hat{u}_{n_{r}}^{(\nu)}}^{\hat{s}}+G_{D, \hat{u}_{n_{r}}^{(\nu)}}^{\hat{s}} \tilde{s}+c \tilde{\delta}^{s}-\tilde{\delta I}_{u}-\tilde{\delta T}_{u}, \\
y_{L 1, \hat{u}_{n_{r}}^{(\nu)}}^{\hat{s}}=\rho_{L 1, \hat{u}_{n_{r}}^{(\nu)}}^{\hat{s}}+G_{D, \hat{u}_{n_{r}}^{(\nu)}}^{\hat{s}}+c \tilde{\delta} t^{s}+\tilde{\delta} I_{u}-\delta \tilde{T}_{u},
\end{gathered}
$$

and

$$
\tilde{C}_{\hat{u}_{1} \cdots \hat{n}_{r}} \equiv\left[\begin{array}{cccccccccc}
1 & G_{\hat{u}_{1}^{(\nu)}} & 1 & & & & & & & \\
1 & G_{\hat{u}_{1}^{(\nu)}} & 1 & I & & & & & & \\
1 & & & & G_{\hat{u}_{2}^{(\nu)}} & 1 & & & & \\
1 & & & & G_{\hat{u}_{2}^{(\nu)}} & 1 & I & & & \\
\vdots & & & & & \ddots & & & & \\
\vdots & & & & & & \ddots & & & \\
1 & & & & & & & G_{\hat{u}_{n}^{(\nu)}} & 1 & \\
1 & & & & & & & G_{\hat{u}_{n r}^{(\nu)}} & 1 & I
\end{array}\right]
$$

or instead of (42), we can write

$$
v \equiv\left[\begin{array}{c}
G_{D, \hat{u}_{1}^{(\nu)}}^{\hat{s}} e_{s}+e_{\delta t^{s}}-e_{\delta I_{u}}-e_{\delta T_{u}}+e_{C A, u_{1}} \\
G_{D, \hat{u}_{1}^{(\nu)}}^{\hat{s}} e_{s}+e_{\delta t^{s}}+e_{\delta I_{u}}-e_{\delta T_{u}}+\lambda_{1} \varepsilon_{L 1, u_{1}} \\
\vdots \\
G_{D, \hat{u}_{n_{r}}^{(\nu)}}^{\hat{s}} e_{s}+e_{\delta t^{s}}-e_{\delta I_{u}}-e_{\delta T_{u}}+e_{C A, u_{n_{r}}} \\
G_{D, \hat{u}_{n_{r}}^{(\nu)}}^{\hat{s}} e_{s}+e_{\delta t^{s}}+e_{\delta I_{u}}-e_{\delta T_{u}}+\lambda_{1} \varepsilon_{L 1, u_{n_{r}}}
\end{array}\right] .
$$

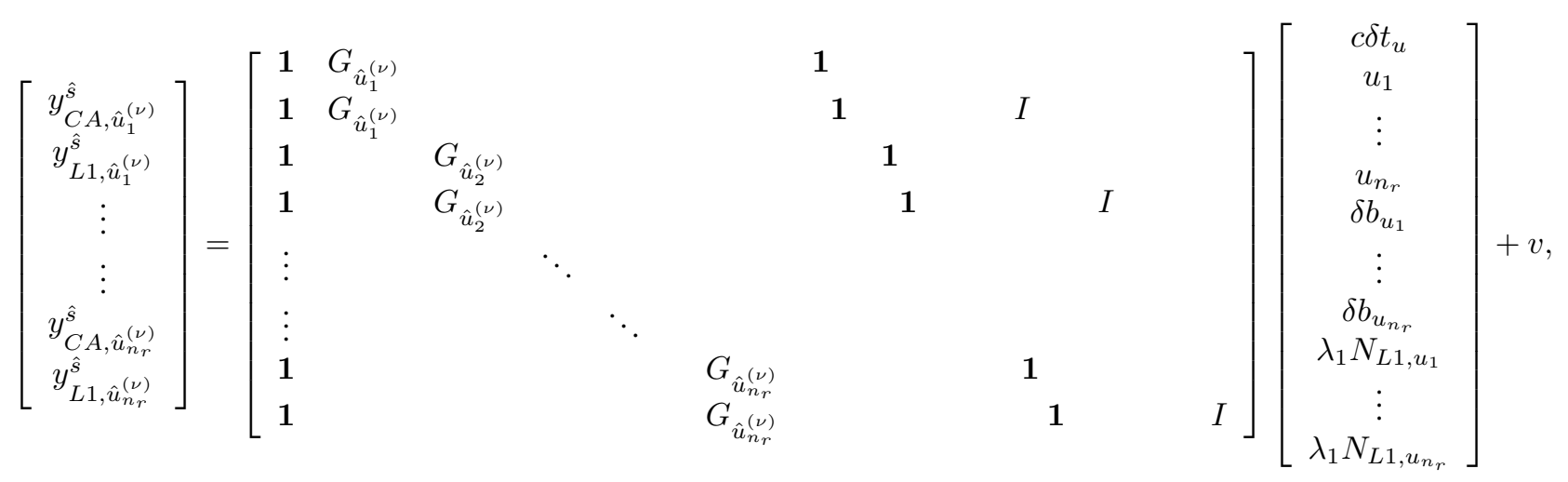


or, we simply express (45) by the vector-matrix form

$$
y_{u_{1} \cdots u_{n_{r}}}=C_{u_{1} \cdots u_{n_{r}}} \theta_{u_{1} \cdots u_{n_{r}}}+v
$$

Also, similary to the first difference idea of GNSS measurements, we subtract each odd row from the first row and each even row from the second, and we neglect first and second block rows such that we have the following relation:

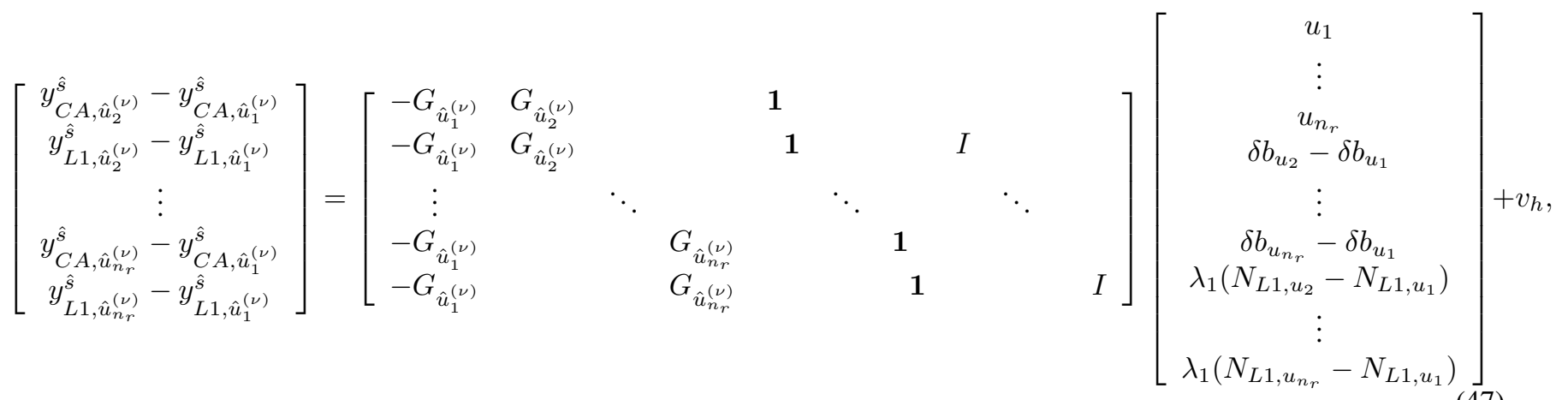

where

$$
v_{h} \equiv\left[\begin{array}{c}
\left(G_{D, \hat{u}_{2}^{(\nu)}}^{\hat{s}}-G_{D, \hat{u}_{1}^{(\nu)}}^{\hat{s}}\right) e_{s}+e_{C A, u_{2}}-e_{C A, u_{1}} \\
\left(G_{D, \hat{u}_{2}^{(\nu)}}^{\hat{s}}-G_{D, \hat{u}_{1}^{(\nu)}}^{\hat{s}}\right) e_{s}+\lambda_{1}\left(\varepsilon_{L 1, u_{2}}-\varepsilon_{L 1, u_{1}}\right) \\
\vdots \\
\left(G_{D, \hat{u}_{n r}^{(\nu)}}^{\hat{s}}-G_{D, \hat{u}_{1}^{(\nu)}}^{\hat{s}}\right) e_{s}+e_{C A, u_{n_{r}}}-e_{C A, u_{1}} \\
\left(G_{D, \hat{u}_{n_{r}}^{(\nu)}}^{\hat{s}}-G_{\left.D, \hat{u}_{1}^{(\nu)}\right)}^{\hat{s}}\right) e_{s}+\lambda_{1}\left(\varepsilon_{L 1, u_{n_{r}}}-\varepsilon_{L 1, u_{1}}\right)
\end{array}\right] .
$$

Then we express approximated relations of the constraints in (41) as follows:

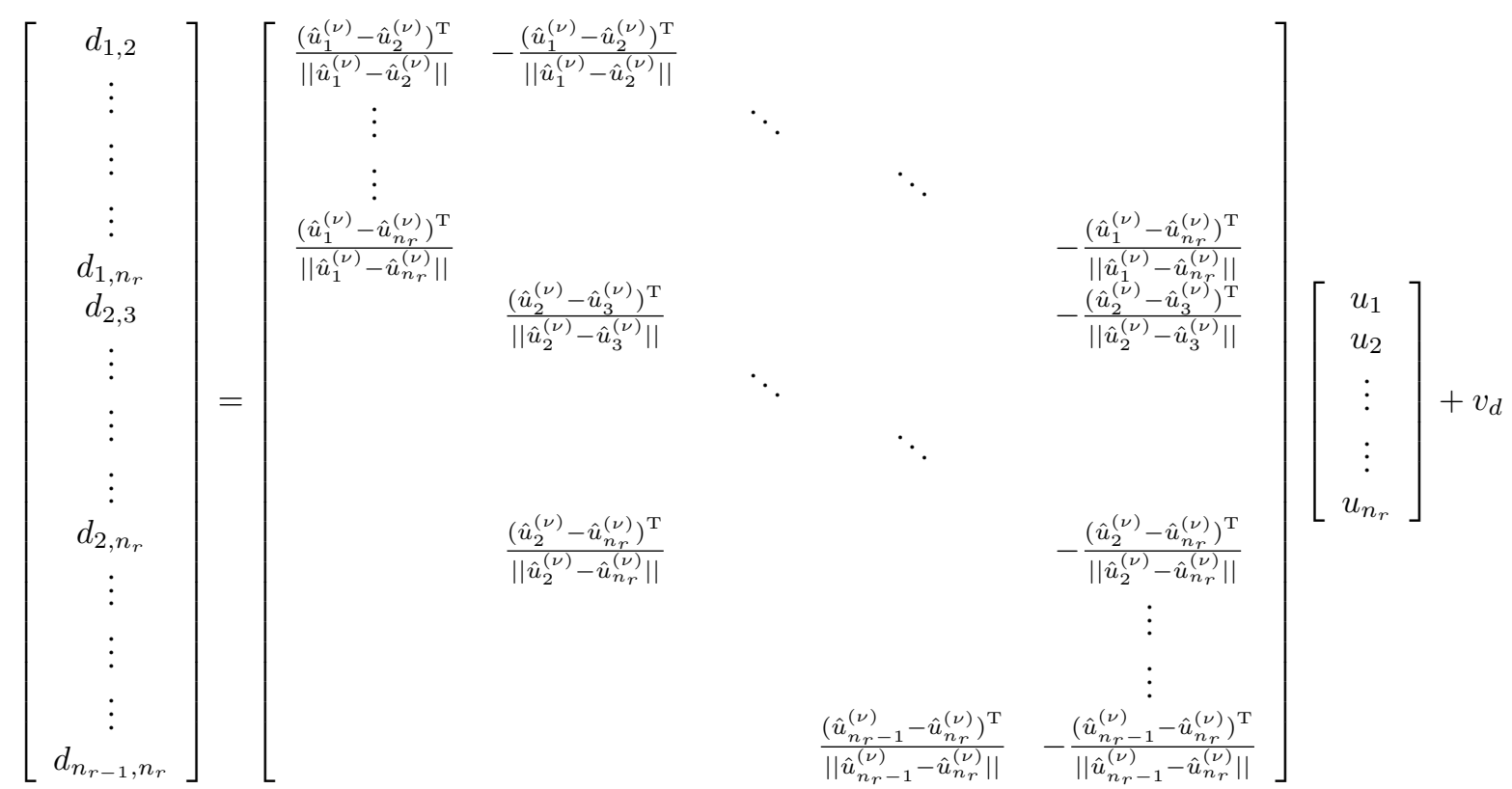

Specially, in the cases of $n_{r}=2,3$, we can write respectively,

$$
\begin{aligned}
& {\left[d_{1,2}\right]=\left[\begin{array}{ll}
\frac{\left(\hat{u}_{1}^{(\nu)}-\hat{u}_{2}^{(\nu)}\right)^{\mathrm{T}}}{\left\|\hat{u}_{1}^{(\nu)}-\hat{u}_{2}^{(\nu)}\right\|} & -\frac{\left(\hat{u}_{1}^{(\nu)}-\hat{u}_{2}^{(\nu)}\right)^{\mathrm{T}}}{\left\|\hat{u}_{1}^{(\nu)}-\hat{u}_{2}^{(\nu)}\right\|}
\end{array}\right]\left[\begin{array}{l}
u_{1} \\
u_{2}
\end{array}\right]+v_{d}} \\
& {\left[\begin{array}{c}
d_{1,2} \\
d_{1,3} \\
d_{2,3}
\end{array}\right]=\left[\begin{array}{ccc}
\frac{\left(\hat{u}_{1}^{(\nu)}-\hat{u}_{2}^{(\nu)}\right)^{\mathrm{T}}}{\left\|\hat{u}_{1}^{(\nu)}-\hat{u}_{2}^{(\nu)}\right\|} & -\frac{\left(\hat{u}_{1}^{(\nu)}-\hat{u}_{2}^{(\nu)}\right)^{\mathrm{T}}}{\left\|\hat{u}_{1}^{(\nu)}-\hat{u}_{2}^{(\nu)}\right\|} & \\
\frac{\left(\hat{u}_{1}^{(\nu)}-\hat{u}_{3}^{(\nu)}\right)^{\mathrm{T}}}{\left\|\hat{u}_{1}^{(\nu)}-\hat{u}_{3}^{(\nu)}\right\|} & & -\frac{\left(\hat{u}_{1}^{(\nu)}-\hat{u}_{3}^{(\nu)}\right)^{\mathrm{T}}}{\left\|\hat{u}_{1}^{(\nu)}-\hat{u}_{3}^{(\nu)}\right\|} \\
& \frac{\left(\hat{u}_{2}^{(\nu)}-\hat{u}_{3}^{(\nu)}\right)^{\mathrm{T}}}{\left\|\hat{u}_{2}^{(\nu)}-\hat{u}_{3}^{(\nu)}\right\|} & -\frac{\left(\hat{u}_{2}^{(\nu)}-\hat{u}_{3}^{(\nu)}\right)^{\mathrm{T}}}{\left\|\hat{u}_{2}^{(\nu)}-\hat{u}_{3}^{(\nu)}\right\|}
\end{array}\right]\left[\begin{array}{l}
u_{1} \\
u_{2} \\
u_{3}
\end{array}\right]+v_{d}}
\end{aligned}
$$




\section{KALMAN FILTERING FOR RTK-VPPP}

Let us show the Kalman filtering algorithm for RTK-VPP positioning based upon the GR equation in (45) and the constrain condition in (49). For this purpose, we discuss and derive the state equations as follows.

\subsection{STATE EQUATIONS}

Let us obtain the state equation for each component of $\theta$ in (45) for RTK positioning.

\subsubsection{State equation for receiver's clock error}

Also the receiver's clock errors are generally modeled as follows [20, 21, 6] (let us call the following model as the A-model of the receiver's clock error),

$$
\begin{aligned}
c \delta t_{u, t+1} & =c \delta t_{u, t}+\Delta_{t} c \dot{\delta} t_{u, t}+w_{c \delta t_{u}, t} \\
c \dot{\delta} t_{u, t+1} & =c \dot{\delta} t_{u, t}+w_{c \dot{\delta} t_{u}, t}
\end{aligned}
$$

where $\Delta_{t}$ denotes the sampling interval of the receiver's clock error, and the noise $w_{c \delta t_{u}, t}$ and $w_{c \dot{\delta} t_{u}, t}$ are assumed as white Gaussian processes with zero means and covariances $q_{c \delta t}$ and $q_{c \dot{\delta} t}$, respectively. Then we can write

$$
\begin{aligned}
{\left[\begin{array}{c}
c \delta t_{u, t+1} \\
c \dot{\delta} t_{u, t+1}
\end{array}\right] } & =\left[\begin{array}{cc}
1 & \Delta_{t} \\
0 & 1
\end{array}\right]\left[\begin{array}{c}
c \delta t_{u, t} \\
\dot{\delta} t_{u, t}
\end{array}\right]+\left[\begin{array}{c}
w_{c \delta t_{u}, t} \\
w_{c \dot{\delta} t_{u}, t}
\end{array}\right] \\
& \equiv F_{A, c \delta t}\left[\begin{array}{c}
c \delta t_{u, t} \\
c \dot{\delta} t_{u, t}
\end{array}\right]+\left[\begin{array}{c}
w_{c \delta t_{u}, t} \\
w_{c \dot{\delta} t_{u}, t}
\end{array}\right]
\end{aligned}
$$

Also we consider another statistical model of the receiver's clock error from the standpoint of time-series analysis. Namely we assume the time derivative of the receiver's clock error is a first order markov process as folows:

$$
\begin{aligned}
& c \delta t_{u, t+1}=c \delta t_{u, t}+\Delta_{t} c \dot{\delta} t_{u, t} \\
& c \dot{\delta} t_{u, t+1}=\kappa c \dot{\delta} t_{u, t}+w_{c \dot{\delta} t_{u}, t}
\end{aligned}
$$

where $\kappa$ denotes the regression coefficient. By the vectormatrix form, we can also describe the model as follows (let call this model as the B-model of the receiver's clock error)

$$
\begin{aligned}
{\left[\begin{array}{c}
c \dot{\delta} t_{u, t+1} \\
c \dot{\delta} t_{u, t+1}
\end{array}\right] } & =\left[\begin{array}{cc}
1 & \Delta_{t} \\
0 & \kappa
\end{array}\right]\left[\begin{array}{c}
c \delta t_{u, t} \\
c \dot{\delta} t_{u, t}
\end{array}\right]+\left[\begin{array}{c}
0 \\
w_{c \dot{\delta} t_{u}, t}
\end{array}\right] \\
& \equiv F_{B, c \delta t}\left[\begin{array}{c}
c \delta t_{u, t} \\
c \dot{\delta} t_{u, t}
\end{array}\right]+\left[\begin{array}{c}
0 \\
w_{c \dot{\delta} t_{u}, t}
\end{array}\right]
\end{aligned}
$$

\subsubsection{State equations for positions}

In the kinematic case, let us show state equations for the positions of the moving objects; $u_{i}, i=1, \cdots, n_{r}$. We often apply one of the dynamical models which are assumed as first-order Markov processes of, the velocity of $u_{i}\left(: v_{i}\right)$, of the acceleration of $u(: a)$ (the so-called Singer's moving model [22]), or of the jerk of $u_{i}\left(: \gamma_{i}\right),[23,24]$, with or without the constraints [25], [26].

For the land vehicle such as automobiles, we adopt Singer's model for the east-west (E) coordinate and the north-south (N) coordinate, and a first order markov model of the velocity for the up-down (U) coordinate, in the local frame (see Fig. 1) [13].

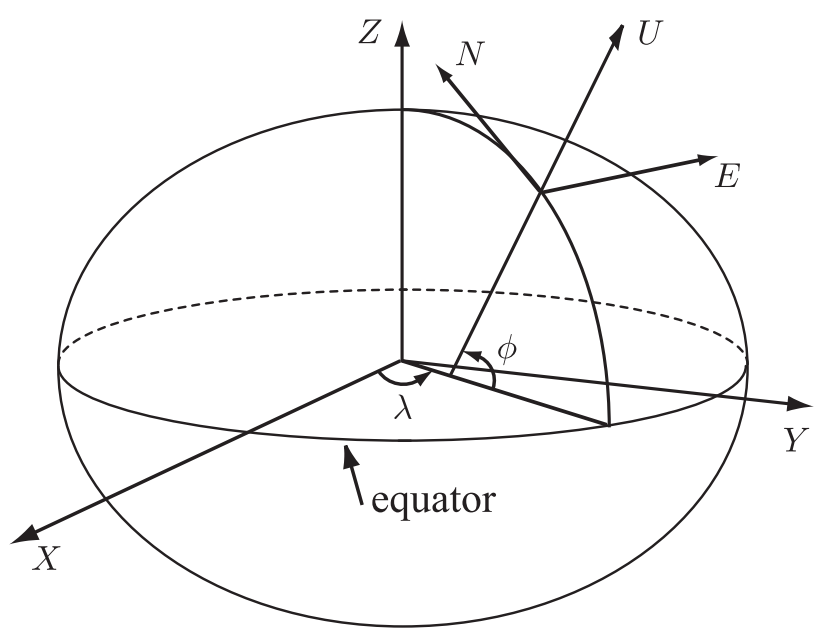

Fig. 1: ECEF-system and ENU-system

where $L$ stands for the coordinates in the local frame (or the local-level system (LLS), or east-north-up (ENU) system) $[18,13]$. Then the transformation matrix to ENU coordinates from WGS-84 coordinates is given by

$T_{i, W}^{L}=\left[\begin{array}{ccc}-\sin \lambda_{i} & \cos \lambda_{i} & 0 \\ -\sin \phi_{i} \cos \lambda_{i} & -\sin \phi_{i} \sin \lambda_{i} & \cos \phi_{i} \\ \cos \phi_{i} \cos \lambda_{i} & \cos \phi_{i} \sin \lambda_{i} & \sin \phi_{i}\end{array}\right]$,

where $\lambda_{i}$ and $\phi_{i}$ denote the longitude and latitude of $u_{i}$, respectively. Let $u_{0}$ in the WGS- 84 coordinate system be an origin in the ENU coordinate system, then any coordinate $u_{i}$ in the WGS-84 system can be described by the ENU system by the relation:

$$
u_{i, L}=T_{i, W}^{L}\left(u_{i}-u_{0}\right) .
$$

Therefore, also we have

$$
u_{i}=u_{0}+\left(T_{i, W}^{L}\right)^{-1} u_{i, L} .
$$

Furthermore by the relation $\left(T_{i, W}^{L}\right)^{-1}=\left(T_{i, W}^{L}\right)^{\mathrm{T}}$, we have

$$
\begin{aligned}
u_{i} \equiv\left[\begin{array}{c}
x_{i} \\
y_{i} \\
z_{i}
\end{array}\right] & =\left[\begin{array}{l}
x_{0} \\
y_{0} \\
z_{0}
\end{array}\right]+\left(T_{i, W}^{L}\right)^{\mathrm{T}}\left[\begin{array}{c}
x_{i, L} \\
y_{i, L} \\
z_{i, L}
\end{array}\right] \\
& =u_{0}+\left(T_{i, W}^{L}\right)^{T} u_{i, L},
\end{aligned}
$$

where

$$
u_{i, L} \equiv\left[x_{i, L}, y_{i, L}, z_{i, L}\right]^{\mathrm{T}} .
$$

Then by the assumption of the Singer's model, namely the accelerations; $a_{x_{i}, L}, a_{y_{i}, L}$, of $x_{i, L}, y_{i, L}$, respectively, are assumed as the first order Markov processes:

$$
\begin{aligned}
& \dot{a}_{x_{i, L}}(t)=-\alpha_{x} a_{x_{i, L}}(t)+w_{a_{x_{i}}}(t) \\
& \dot{a}_{y_{i, L}}(t)=-\alpha_{y} a_{y_{i, L}}(t)+w_{a_{y_{i}}}(t) .
\end{aligned}
$$


Also the velocity of $v_{z_{i}, L}$ of $z_{i, L}$ is assume as a first order Markov process:

$$
\dot{v}_{z_{i, L}}(t)=-\alpha_{z} v_{z_{i, L}}(t)+w_{v_{z_{i}}}(t)
$$

Then, we have the following form of the discrete-time state equation [13] for $u_{E, i, L}$ :

$$
u_{E, i, L, t+1}=\tilde{A}_{i, t} u_{E, i, L, t}+\tilde{B}_{i, t} w_{i, t}
$$

where

$$
u_{E, i, L} \equiv\left[\begin{array}{c}
x_{i, L} \\
y_{i, L} \\
z_{i, L} \\
v_{x_{i}, L} \\
v_{y_{i}, L} \\
v_{z_{i}, L} \\
a_{x_{i}, L} \\
a_{y_{i}, L}
\end{array}\right]
$$

Furthermore, we use the following state equations for $\delta b_{u_{i}}$ and $N_{L 1, u_{i}}$ for $i=1, \cdots, n_{r}$ :

$$
\begin{aligned}
\delta b_{u_{i}, t+1} & =\delta b_{u_{i}, t}, \\
N_{L 1, u_{i}, t+1} & =N_{L 1, u_{i}, t} .
\end{aligned}
$$

Collecting all state equations in (54) or (56), (64), (66), and (67), we finally have the following state equation:

$$
\eta_{L, t+1}=A_{t} \eta_{L, t}+B_{t} w_{t}
$$

where

$$
\eta_{L} \equiv\left[\begin{array}{c}
c \delta t_{u} \\
c \dot{\delta} t_{u} \\
u_{E, 1, L} \\
\vdots \\
u_{E, n_{r}, L} \\
\delta b_{u_{1}} \\
\vdots \\
\delta b_{u_{n_{r}}} \\
\lambda_{1} N_{L 1, u_{1}} \\
\vdots \\
\lambda_{n_{r}} N_{L 1, u_{n_{r}}}
\end{array}\right]
$$

Then the observation equation in (46) should be changed by applying the relations in (60), (65) and the constraints in (49).

\section{Altitude Information}

Further, in urban canyons, due to a few GNSS satellite observables, it is quite useful for precise positioning to have the altitude information of the user's position as

$$
\hat{z}_{i, L}=z_{i, L}+e_{z_{i, L}}
$$

where $z_{i, L}$ is the $i$-th user's altitude in the local frame (or the local-level system (LLS) or east-north-up(ENU) system), where we had discussed the dynamical models of automobiles in the ENU system and the WGS-84 system. Then the measurement equation is again changed to

$$
y_{t}=C_{t} \eta_{L, t}+v_{t},
$$

Therefore the positioning algorithms based on the Kalman filter for (68) and (71) are given as follows [12, 13]:

$$
\begin{array}{rlrl}
\hat{\eta}_{L, t+1 \mid t} & =A_{t} \hat{\eta}_{L, t \mid t} \\
\hat{\eta}_{L, t \mid t} & =\hat{\eta}_{L, t \mid t-1}+K_{t} \nu_{t} \\
\nu_{t} & \equiv & y_{t}-C_{t} \hat{\eta}_{L, t \mid t-1} & \multicolumn{1}{c}{(72)} \\
K_{t} & = & \Sigma_{t \mid t-1} C_{t}^{\mathrm{T}}\left[C_{t} \times \Sigma_{t \mid t-1} C_{t}^{\mathrm{T}}+R_{t}\right]^{-1} & (75) \\
\Sigma_{t+1 \mid t} & = & A_{t} \Sigma_{t \mid t} A_{t}^{\mathrm{T}}+Q_{t} \\
\Sigma_{t \mid t} & = & \Sigma_{t \mid t-1} \\
& & -K_{t} C_{t} \Sigma_{t \mid t-1} \\
& & \text { Init. Cond.: }\left\{\begin{array}{l}
\hat{\eta}_{L, 0 \mid-1}=\bar{\eta}_{L, 0} \\
\Sigma_{0 \mid-1}=\Sigma_{0}
\end{array}\right.
\end{array}
$$

\section{Remark: Another use of constraints}

Another estimation method of $u_{i} ; i=1, \cdots, n_{r}$ for using the cnstraints in (49). Namely, at first, we can obtain the estimates $\hat{u}_{i} ; i=1, \cdots, n_{r}$ by Kalman filtering to (45) without using the constarints in (49). After assuming the unbiased estimates $\hat{u}_{i} ; i=1, \cdots, n_{r}$ and their Gaussian statistics:

$$
\begin{aligned}
\mathrm{E}\left[\hat{u}_{1,2, \ldots, n_{r}}\right] & =u_{1,2, \ldots, n_{r},} \\
\operatorname{Var}\left[\hat{u}_{1,2, \ldots, n_{r}}\right] & =R_{1,2, \ldots, n_{r}},
\end{aligned}
$$

where $u_{1,2, \ldots, n_{r}} \equiv\left[u_{1}^{\mathrm{T}}, u_{2}^{\mathrm{T}}, \cdots, u_{n_{r}}^{\mathrm{T}}\right]^{\mathrm{T}}, \hat{u}_{1,2, \ldots, n_{r}} \equiv$ $\left[\hat{u}_{1}^{\mathrm{T}}, \hat{u}_{2}^{\mathrm{T}}, \cdots, \hat{u}_{n_{r}}^{\mathrm{T}}\right]^{\mathrm{T}}$. Now, consider the followng relation for the conditional probability function

$$
p_{U \mid D}\left(u_{1, \cdots, n_{r}} \mid d\right)=\frac{p_{U, D}\left(u_{1, \cdots, n_{r}}, d\right)}{p_{D}(d)},
$$

where $d=\left[d_{1,2}, \cdots, d_{n_{r-1}, n_{r}}\right]$ in (49) for notational simplicity. Then we can obtain the Maximum A Posterior (MAP) estimates [28] by evaluating [2]

$$
\begin{aligned}
& \arg \max _{u_{1, \cdots, n_{r}}} p_{U \mid D}\left(u_{1, \cdots, n_{r}} \mid d\right) \\
= & \arg \max _{u_{1, \cdots, n_{r}}} \frac{p_{U, D}\left(u_{1, \cdots, n_{r}}, d\right)}{p_{D}(d)} \\
= & \arg \max _{u_{1, \cdots, n_{r}}} p_{U, D}\left(u_{1, \cdots, n_{r}}, d\right) .
\end{aligned}
$$

\section{EXPERIMENTAL RESULTS}

Here, in preliminary positioning experiment of VPPP are carried out by using the Kalman filter formulation for static positioning case not for kinematic positioning. In the experiments, Millennium GPSCard (OEM3), PROPAK BeeLine GPSCard (OEM3), GPS-600 antenna and Active GPS501 antenna of NovAtel Inc. were located (fixed) on the 
roof of the 7 stories West-Wing Building at Ritsumeikan Biwako-Kusatsu Campus (BKC), Shiga, Japan. Prior to the experiments, the coordinates of the GPS-600 antenna position were precisely obtained by the carrier phase differential positioning method applied by the GPS observation data collected by the GPS Earth Observation Network (GEONET) of the Geographical Survey Institute (GSI) in Japan. The result of the carrier phase differential positioning is shown in Table 1, and it is expected to be within an accuracy of several $[\mathrm{mm}]$ to $1[\mathrm{~cm}]$. Therefore this result is regarded as true for evaluating the following VPPP experimental results.

Table 1: Result of carrier phase differential positioning (assumed to be true) in WGS-84

\begin{tabular}{|c|c|c|}
\hline Lat.[deg.] & Lon.[deg.] & Ell. Height[m] \\
\hline 34.98187600 & 135.96409800 & 224.177 \\
\hline
\end{tabular}

In order to apply the Kalman filter to Eq. (45), the covariance matrices of the observation noise $v$ is respectively calculated from the general standard deviation of the each error source shown in [29]. Also the receiver was fixed in this experiments, therefore the each component of the state vector is constant.

For the experiment, a total of 2160 epochs single frequency data were collected at $0.2[\mathrm{~Hz}]$ rate on January 26, 2006, from 18:00'00 to 22:00'00 UTC. The number of visible satellites was between 5 to 8 during the test periods.

Fig. 1 shows the positioning errors with the local level axes (East, North and Height (Up) ), where the dashed line shows the error when using two PPP results and their arithmetic averages(AA) and the solid line shows the error when using VPPP with Eq. (45). Fig. 2 shows the positioning errors in the horizontal N-E plane, where the 'triangle' symbols shows the error when using PPP (Arithmetic Ave.) and the 'circle' symbols shows the error when using VPPP with Eq. (45). The errors were computed by differencing the estimated position from the coordinates of Table 1. We can observe from Figs. 1 and 2 that the positioning quality is considerably improved by using VPPP. The statistics of the positioning results are summarized in Table 2 .

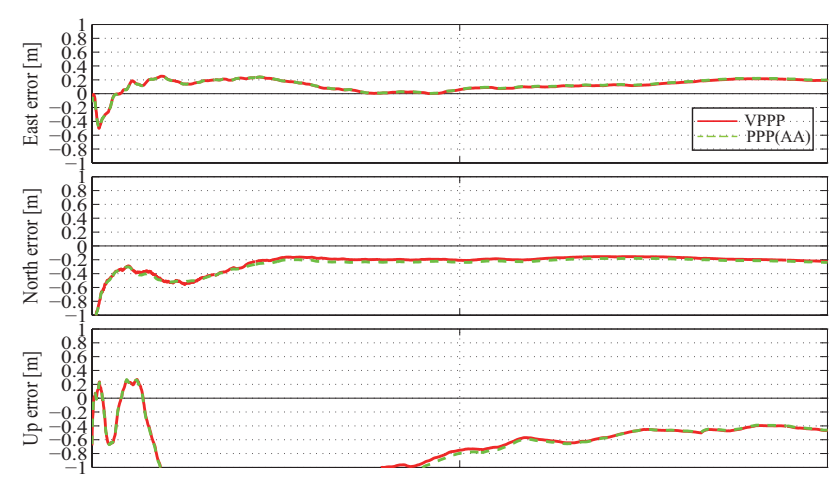

Fig. 1: North, East and Up error (Jan. 26, 2006)

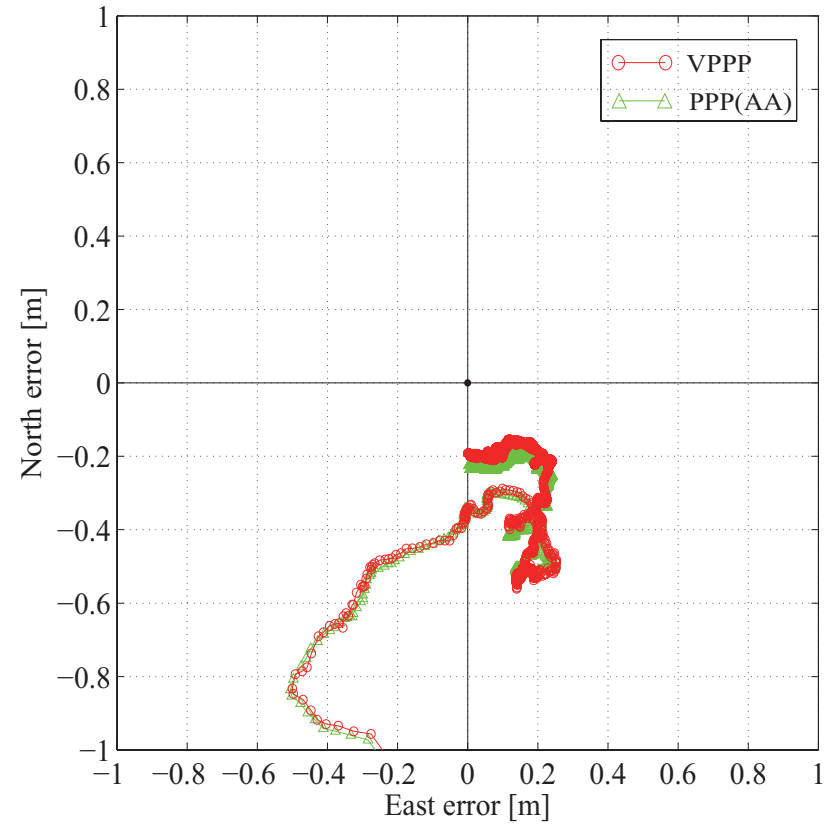

Fig. 2: Horizontal error (Jan. 26, 2006)

Table 2: Summary statistics of VPPP results (Jan. 26, 2006)

\begin{tabular}{|c|c|c|c|c|}
\hline Dataset & Dir. & Bias[m] & Std. Dev.[m] & RMS[m] \\
\hline \hline \multirow{4}{*}{ PPP (AA) } & $\mathrm{E}$ & 0.1224 & 0.0989 & 0.1574 \\
\cline { 2 - 5 } & $\mathrm{N}$ & -0.2684 & 0.1296 & 0.2980 \\
\cline { 2 - 5 } & $\mathrm{U}$ & -0.8199 & 0.4859 & 0.9530 \\
\cline { 2 - 5 } & Horiz. & 0.2950 & 0.1630 & 0.3370 \\
\hline \hline \multirow{4}{*}{ VPPP } & $\mathrm{E}$ & 0.1196 & 0.0978 & 0.1545 \\
\cline { 2 - 5 } & $\mathrm{N}$ & -0.2445 & 0.1372 & 0.2804 \\
\cline { 2 - 5 } & $\mathrm{U}$ & -0.7955 & 0.4666 & 0.9222 \\
\cline { 2 - 5 } & Horiz. & 0.2722 & 0.1685 & 0.3201 \\
\hline
\end{tabular}

\section{CONCLUSIONS}

We presented new GR equations that contain the effect of the receiver's hardware biases as well as the satellite's hardware biases to the previous GR models for GNSS positioning algorithms. Applying the coupled GR equations for multiple antennas in the case of unknown positions, we derive a new very precise point positioning (VPPP). And the experiments in the static situation were carried out. As a result, the VPPP algorithm could give the positioning accuracy in decimeter level and achieve higher accuracy than PPP algorithm. In the future research, we plan to examine the algorithm in the kinematic environment.

\section{REFERENCES}

[1] S. Sugimoto, Y. Kubo, S. Fujita: Very Precise Point Positioning Based on GR Models, Proc. of the 38th ISCIE International Symposium on Stochastic Systems Theory and Its Applications, Nagano, Japan, Nov. (2006). 
[2] S. Sugimoto, Y. Kubo and S. Fujita: Carrier-Phase-Based PPP with Multiple Antennas Based on GR Models, Proc. ION National Technical Meeting, pp. 881-891, San Diego, California, Jan. (2007).

[3] S. Fujita, Y. Kubo and S. Sugimoto: Further Study on PPP Based on GR Models with Multiple Antennas, Int. J. of Innovative Computing, Information and Control, vol. 4, no. 1, pp. 127-141, Feb. (2008).

[4] M. Kamimura, R. Tomita, T. Nagano, A. Chabata, Y. Kubo and S. Sugimoto: Detection of Cycle Slips and Multipath in GNSS RTK Precise Point Positioning, Proc. ION-GNSS 2011, Portland, Oregon, pp. 1056-1067, Sept. (2011).

[5] A. Chabata, M. Kamimura, Y. Kubo and S. Sugimoto: Detection and Exclusion of Abnormal Measurement Data in GNSS Precise Point Positioning, Proc. of the 43rd ISCIE International Symposium on Stochastic Systems Theory and Its Applications, Shiga, pp. 119-126, March (2012).

[6] A. Chabata, Y. Suzuki, Y. Kubo and S. Sugimoto: RTK-PPP Algorithms using GNSS Observables from Few Satellites, Proc. ION-GNSS 2012, Nashville, Tennessee Sept. (2012).

[7] S. Sugimoto and Y. Kubo: GNSS Regressive Models and Precise Point Positioning, Proc. of the 36th ISCIE Int. Symp. on Stochastic Systems Theory and Its Applications, pp. 159 164, Saitama, Nov. (2004).

[8] S. Sugimoto and Y. Kubo: Carrier-Phase-Based Precise Point Positioning - a Novel Approach Based on GNSS Regression Models, Proc. of 2004 International Symposium on GPS/GNSS, Sydney, Dec. (2004).

[9] Y. Kubo, A. Kitao, S. Fujita, and S. Sugimoto: A New RTK Algorithm for Carrier-Phase-Based Precise Point Positioning Based on GNSS Regression Model, Proc. ION-GNSS 2005, pp. 1492-1499, Long Beach Sept. (2005).

[10] S. Fujita, Y. Kubo and S. Sugimoto: Precise Point Positioning based on GR Models - Further Results in Single Frequency Applications Proc. IGNSS Society 2006 Symposium, Paper No. 92, Queensland, Australia, July (2006).

[11] S. Fujita, Y. Kubo and S. Sugimoto: A Single Frequency PPP Algorithm Based on GR Models Proc. ION GNSS 2006 Symposium, Fort Worth, Texas, Sept. (2006).

[12] S. Sugimoto and Y. Kubo: Unified Methods of Point and Relative Positioning Based on GNSS Regression Equations Proc. ION GNSS 2006 Symposium, Fort Worth, Texas, Sept. (2006).

[13] S. Sugimoto: GNSS Positioning Algorithms based on GR Models, Section 7.3.1 of GPS Handbook (in Japanese) ed. by S. Sugimoto and R. Shibasaki, Asakura-Shoten, Tokyo, Japan (2010).

[14] Y. Gao, and X. Shen: A New Method for Carrier-PhaseBased Precise Point Positioning, Journal of the Institute of Navigation, Vol 49, No. 2, pp. 109-116 (Summer 2002).
[15] Y. Gao and Z. Z. Lin: Precise Ionosphere Modeling Using Regional GPS Network Data, J. of Global Positioning Systems, Vol. 1, No. 1, pp. 18-24 (2002).

[16] P. J. G. Teunissen and A. Kleusberg (Eds.): GPS for Geodesy, 2nd Edition, Springer-Verlag, New York (1998).

[17] A. Leick: GPS Satellite Surveying, 3rd Edition, John Wiley \& Sons, New York (2004).

[18] P. Misra and P. Enge: Global Positioning System -Signals, Measurements, and Performance, Ganga-Jamuna Press, Massachusetts (2001).

[19] B. Hofmann-Wellenhof, H. Lichtenegger and J. Collins: GPS; Theory and Practice, 5th revised edition, SpringerVerlag (2001).

[20] R. G. Brown and P. Y. C. Hwang: Introduction to Random Signals and Applied Kalman Filtering, 3rd Edition, John Wiley \& Sons, New York (1997).

[21] B. W. Parkinson and J. J. Spilker: The Global Positioning System: Theory and Applications, Vol. I, AIAA Publications (1996).

[22] R. A. Singer: Estimating Optimal Tracking Filter Performance for Manned Maneuvering Targets, IEEE Trans. Aerospace and Electronic Systems, Vol. AES-6, No. 4, pp. 473-483 (1970).

[23] T. Aoki, Y. Shimogaki, T. Ikki, M. Tanikawara, S. Sugimoto, Y. Kubo and K. Fujimoto: Cycle Slip Detection in Kinematic GPS with a Jerk Model for Land Vehicles, Int. J. of Innovative Computing, Information and Control, vol. 5, no. 1, pp. 153-166, January (2009).

[24] T. Aoki and S. Sugimoto: Dynamical Models for Automobile Movements, Int. J. of Innovative Computing, Information and Control, vol. 6, no. 1, pp. 3-14, January (2009)

[25] C. Uratani, K. Sone, Y. Muto, S. Maruo, and S. Sugimoto: A New Method for Carrier-Phase-Based Precise Point, Proc. 16th Int. Technical Meeting of the Satellite Division of the Institute of Navigation (ION GPS/GNSS 2003), pp. 809-818, Portland, OR, Sept. (2003).

[26] Y. Muto, Y. Kubo, Y. Kubo and S. Sugimoto: New Dynamical Models for Kinematic GPS Positioning, Proc. 17th Int. Technical Meeting of the Satellite Division of the Institute of Navigation (ION GNSS 2004), Long Beach, CA, Sept. (2004).

[27] Y. Kubo, Y. Suzuki, M. Ozaki, M. Ohashi and S. Sugimoto: Methods for Improving PPP Accuracy in Urban Canyons, Proc. ION-GNSS 2013, Nashville, Tennessee, Sept., (2013).

[28] Harry L. Van Trees: Detection, Estimation, and Modulation Theory, Part 1, John Wiley \& Sons, New York (2001).

[29] J. B.-Y. Tsui: Fundamentals of Global Positioning System Receivers A Software Approach, John Wiley \& Sons, New York (2000). 